\title{
Moving to Diversity
}

\section{Residential Mobility, Changes in Ethnic Diversity, and Concerns About Immigration}

\author{
Bram Lancee \\ Utrecht University, the Netherlands
}

Merlin Schaeffer

University of Cologne, Germany

Please cite as: Lancee, B., \& Schaeffer, M. (2015). Moving to diversity. Residential mobility, changes in ethnic diversity, and concerns about immigration. In R. Koopmans, B. Lancee, \& M. Schaeffer (Eds.), Social Cohesion and Immigration in Europe and North America: Mechanisms, Conditions, and Causality (pp. 38-55). London: Routledge.

\begin{abstract}
Studies on ethnic diversity and social cohesion are predominantly cross-sectional. Relying on longitudinal data from the German Socio-Economic Panel and applying a differences-in-differences design, this paper investigates how the event of moving to a more or less diverse neighbourhood affects people's opinions about immigration up. This longitudinal design not only excludes reverse causality, but also renders unobserved heterogeneity a very unlikely alternative explanation. We show that individuals who move to a more diverse neighborhood are about $13 \%$ more likely to become very concerned about immigration. This effect remains even three years after the move. Because individuals who move to equal or lower diversity neighborhoods do not change their attitudes, it is not the event of moving as such that makes people more negative towards out-groups. Moreover, this study is the first to investigate whether previous experiences with diversity eliminate any threat effects of moving to a more diverse place. Interestingly, our results lend only partial support for this hypothesis, since those who to the top 15 per cent most diverse neighborhoods generally do not change their views. We consider whether this particular null-effect might be due to 'threat immunity' of such movers. Our sensitivity analyses questions this objection and instead suggests that populations often thought to be immune to threat seem to respond similarly.
\end{abstract}




\section{Introduction}

While the amount of studies that analyze the relation between ethnic diversity and social cohesion is burgeoning (Koopmans, Lancee, \& Schaeffer 2015), there is virtually no empirical research that relies on longitudinal data. Moreover, the few longitudinal studies that are available do not analyze individuals over time but rely on repeated cross-sections of countries (Meuleman, Davidov, \& Billiet, 2009), municipalities (Dinesen \& Sønderskov, 2012) or panels of municipalities (Hopkins, 2009). The scarcity of dynamic studies is problematic for several reasons, since the questions underlying the relation between diversity and trust are often more fruitfully put in a longitudinal perspective. For some, longitudinal analysis allows to better deal with concerns about causality. As Hémet (2015), Fisher Williamson (2015), and Veit (2015) demonstrate impressively, such concerns can also be tackled using other methods. In this paper, we demonstrate that a longitudinal perspective also provides substantial insights. As Hopkins (2009, p. 160) states: "To understand how diversity influences public good provision, we should look to those towns that are diversifying, not those towns that are diverse." Or, put differently, the guiding question in the debate is how changes in ethnic diversity affect changes in trust and social cohesion.

Yet, such a question can hardly be solved satisfactorily with cross-sectional data (Jackson \& Mare, 2007). Improving upon purely cross-sectional work, some studies show relations between changes in diversity or recent increases therein and (crosssectional) levels of xenophobia (Hopkins, 2010) or social cohesion (Schaeffer, 2014). Still, these studies face the problem of cross-sectional analysis that it is very difficult to empirically separate cause and effect, because there is only one time point measured. In this paper, we use longitudinal data to analyze residential mobility across neighborhoods with different levels of ethnic diversity. By modeling moving house transitions, we analyze how changes in ethnic diversity affect changes in people's attitudes towards immigrants. Such attitudes are not orthodox indicators of social cohesion, such as social trust or civic engagement. Yet, sentiments towards ethnic minorities, also referred to as xenophobia, are an important obstacle to cohesion in any immigration country. Along the same line of reasoning, Uslaner (2015) argues that positive inter-group attitudes are an important pre-condition for more general levels of social trust. Irrespective of the particular indicator of social cohesion, one should keep in mind that a change in diversity because of moving is not the same as a changing 
neighborhood composition. For example, if the diversity of one's neighborhood increases twofold within a year, this is probably a more extreme experience than moving to a neighborhood that is twice as diverse.

Besides its potential for causal analysis, another great advantage of using longitudinal data is that it allows for answering different questions. For example, one can model transitions and thus differentiate between origin and destination. Larger shares of the immigrant population have been argued and found to be both positively (via intergroup contact) and negatively (via group threat) correlated with anti-immigrant attitudes simultaneously (Dixon, 2006; Schlueter \& Scheepers, 2010; Schlueter \& Wagner, 2008). Such opposite findings could potentially be explained by the nature of where someone came from, i.e. the origin category. It is likely that how a change in diversity matters depends on previous experiences with diversity. It could be that increasing diversity is threatening for those who previously lived in homogeneity, because little intergroup contact is made in homogenous areas. On the other hand, individuals moving from diversity to very high diversity are more accustomed to diversity and might see the increase in diversity as an opportunity for further intergroup contact (Martinovic, van Tubergen, \& Maas, 2009). However, there is virtually no research that differentiates origin categories.

Also with regard to the destination of one's move one can expect different effects on xenophobia. In that case, different destinations imply different effects. For example, Schneider (2008) and Schaeffer (2013) find a non-linear relationship between outgroup size and perceived ethnic threat, which suggests that threat effects level off. Yet, there is no previous research that analyzes whether moving to a neighborhood with a very high level of diversity has different effects than moving to a moderately diverse neighborhood.

A methodological advantage of longitudinal analysis is that one can better deal with the problem of potential bias due to selection. When the ethnic composition of a neighborhood changes, better-situated people tend to move to other areas, leaving behind the deprived, low-trusting, and prejudiced inhabitants (Clark, 1992; Crowder, 2000; Crowder, Hall, \& Tolnay, 2011; Harris, 2001). Conversely, prejudice determines the choice for a more homogenous destination neighborhood (Bobo \& Zubrinsky, 1996; Eric Oliver \& Wong, 2003), which would imply that, over time, homogenous neighborhoods not only become more homogenous, but their inhabitants 
also more prejudiced. As Gundelach and Freitag (2013, p. 16) put it: “Affluent and high-trusting residents chose to live in homogeneous neighborhoods. Regarding the choices of residents with fewer financial resources, self-selection could mean that minority members predominantly self-select into neighborhoods where the leasttrusting residents live".

The contribution of this paper is fourfold. First, by analyzing changes in ethnic diversity and attitudes toward immigrants, we better test the causal path that is assumed in the theory. Second, by defining specific transitions, we take both origin and destination into account. Third, we estimate the effect of moving to diversity over time and see whether the reaction to diversity wears off and how long it takes to do so. Fourth, our empirical method allows us to better deal with potential bias due to unobserved heterogeneity and selection.

We make use of the German Socio-Economic Panel Survey (SOEP) and detailed neighborhood data. Applying a differences-in-differences design, we use moving as a 'treatment' of changing diversity and calculate changes in people's attitudes to immigration over multiple years after they have moved. The DID-design controls for all unobserved heterogeneity between individuals, which enables us to isolate the effect of moving from possible confounding factors. Furthermore, in our DID-design we model the causal ordering as we assume it in the theory. This is a big improvement over, for example, regular fixed-effects models

\section{Ethnic diversity and attitudes towards immigrants - longitudinal ev- idence}

As outlined by Koopmans, Lancee and Schaeffer (2015), there is a substantial body of (cross-sectional) work that examines the relation between diversity and attitudes towards immigrants. Yet, longitudinal evidence is rare. One of the few longitudinal studies analyzing out-group size is the work of Meuleman, Davidov and Billiet (2009). They relate changes in migration flows on the country level to changes in attitudes towards immigrants and find that higher inflow is related to more negative attitudes. Lancee and Pardos-Prado (2013) use individual-level longitudinal data in Germany to explain attitudes towards immigrants. They find that the proportion of foreigners predicts attitudes towards immigrants. However, this effect is no longer significant when 
using fixed-effects estimation, suggesting that the effect is explained by unobserved variables or only occurs after a longer period of time. Dinesen and Sønderskov (2012) use longitudinal data (in the form of repeated cross-sections) to predict the effect of ethnic diversity (operationalized as out-group size) on generalized trust in municipalities in Denmark. While trust is not the same as attitudes towards immigration, Dinesen and Sønderskov show, using fixed-effects estimation, that diversity negatively predicts generalized trust, suggesting a group threat mechanism. As we will outline below, our aim in this paper is to go a step beyond merely testing the relation between diversity and cohesion longitudinally.

\section{Moving to diversity: theoretical expectations}

Studies that analyze residential mobility show that moving is selective. For example, supporting the 'White flight hypothesis', in the US the likelihood of leaving the neighborhood increases significantly with the size of and the increase in the minority population (Crowder, 2000; Crowder et al., 2011). Bobo and Zubrinsky (1996) conclude that pre-move levels of prejudice determine the preference and choice for a more homogenous destination neighborhood. Yet, while there is much research that analyzes why and which people move, studies that analyze the effect of moving itself are scarce.

What then, can we expect of the effect of moving? In general, residential mobility is an 'interrupting' event, i.e. it pulls people out of their networks and everyday life. As such, it is associated with lower well-being (Stokols \& Shumaker, 1982). More specifically, residential mobility is said to have both psychological and attitudinal consequences (Lin, Twisk, \& Rong, 2011; Oishi, 2010). Moving is associated with the 'personal' over the 'collective self' and with duty-free friendships and group memberships, rather than duty-based friendships (Oishi, 2010). In line with this, it is also found that residential mobility decreases (behavioral) social capital (de Souza Briggs, 1998; Nisic \& Petermann, 2013; Pettit \& McLanahan, 2003). Furthermore, residential stability is related to more pro-social community and helping behavior (Oishi et al., 2007). It is also often reported that longer duration of residence is associated with more neighbor-interaction, close-knit networks of local ties, and local social capital (Kang \& Kwak, 2003; Sampson, 1988). As moving interrupts people's lives, it might 
very well make one particularly prone to perceptions of threat, including out-group threat.

The question is, however, whether particular moves affect people's inter-group attitudes differently. When moving implies changing diversity, it is likely that intergroup conflict and contact mechanisms operate. The question is which mechanism dominates. That is, ceteris paribus, does a change in ethnic diversity result in positive, negative, or no changes in attitudes towards immigrants? Although not studying individual-level changes, previous work that analyzed changes in diversity dominantly supports the group-threat mechanism explaining attitudes towards immigrants (Lancee \& Pardos-Prado, 2013; Meuleman et al., 2009) and social trust (Dinesen \& Sønderskov, 2012). As diversity increases, people experience (or perceive) the larger out-group size as a threat to their interests, identity, and community. It seems likely that a move to increasing diversity results in more anti-immigrant sentiment.

We should also acknowledge that moving is surely a disruptive, but not an accidental event. People plan to move and thus know where they will live in the future. In other words, because people imagine how their future life will be there could be anticipation effects. Insofar as group threat is one of the mechanisms explaining negative diversity effects, it could be that we observe increasing concerns about immigration already before people move. In other words, once people know that they are going to move to a more diverse neighborhood, the outlook of increased competition over resources and conflicting ways of life might start to threaten them, resulting in increasing concerns prior to the event of moving itself.

A related question is how threat develops after the actual move. Both increasing and decreasing effects are theoretically plausible. Because of familiarization (Schneider (2008) threat could decline over time. To the extent that threat is reflected in real competition over scare resources (Olzak, 1992), threat could also stabilize or even increase. As a general hypothesis, we therefore expect that moving to a more diverse neighborhood causes negative attitudes towards immigrants after, but also shortly before the event. It is an open question how this effect develops after people have moved.

However, this does not necessarily imply that when diversity decreases, people become less xenophobic, too. Certainly, increases in out-group size or diversity trigger 
perceptions of threat, due to fear of job-loss and increasing housing prices. One could argue that decreases in out-group size result in more relaxed views on competition over scarce resources on the labor market or housing market. However, we do not know whether people who have experienced diversity and have become concerned about immigration as a consequence, 'forget' their concerns once they move to homogeneity. Put differently, to the extent that people move to more homogenous neighborhoods because they hold prejudiced attitudes (Bobo \& Zubrinsky, 1996), it is the question whether such individuals change their mind about this contentious political issue, once they have fewer intergroup contact on an everyday basis. We thus refrain from formulating a general hypothesis on the consequences of moving to homogeneity and treat this aspect of the analysis as exploratory.

\section{Origin and destination}

It is plausible that previous experiences with diversity matter for the effect of moving to diversity on attitudes towards immigrants. In other words, there could be an origin effect. Schlueter and colleagues $(2010 ; 2008)$ conclude that perceived out-group size increases perceived group threat, which in turn is related to anti-immigrant attitudes. Semyonov and colleagues (2004) find that, in Germany, higher perceived, but not actual group size is related to anti-immigrant attitudes. If the perception of out-group size matters for people's feelings of threat, it is likely that these perceptions differ for people with and without experience of diversity. However, there are no studies that differentiate previous from current experience with diversity. We propose that while perceived group threat increases after moving to diversity, this increase is stronger for people without 'experience' with diversity, i.e., those who lived in homogenous neighborhoods before moving. In contrast, people who move from a diverse to a highly diverse neighborhood have experience with diversity and are therefore less likely to "over-perceive" the out-group sizes; consequently, out-group threat is lower. We therefore hypothesize that the effect of moving from diversity is different from that of moving from homogeneity.

Similarly, the destination might matter: Moving to high diversity may be different from moving to diversity. As Semyonov Raijman and Gorodzeisky (2006, p. 430) put it: 'Because hostility, prejudice, and discriminatory views are constrained by a certain 
threshold (a ceiling effect), we expect negative views toward outsiders to increase at a faster rate early on, but then to stabilize'. Yet, Semyonov and colleagues do not find a curvilinear effect. Schneider (2008) does find (across countries) a non-linear relationship between out-group size and perceived ethnic threat. Schneider labels this the 'familiarization hypothesis': because of more experience with immigration and integration there is a 'getting used to effect': at some point, a higher percentage of immigrants does not increase anti-immigrant attitudes anymore. Also Schaeffer (2013) finds that, in Germany, the odds of using ethnic categories to describe neighborhood problems rises with larger out-group size. However, at very high levels of foreign nationals in the neighborhood, this effect levels off and the odds even decrease. Aside from familiarization, an explanation for non-linear effects of diversity could be that at high levels of diversity opportunities for contact are pervasive and cancel out ethnic threat. It has repeatedly been found that inter-group contact (such as inter-ethnic friendships and intermarriages) increases with the percentage of ethnic minorities, because the opportunities for contact rise (Martinovic et al., 2009). Yet, the non-linear effect reported by Schneider (2008) controls for having immigrant friends and colleagues, suggesting that familiarization with immigrants in the form of weak intergroup neighborhood acquaintances or encounters explain differential effects of ethnic diversity (Koopmans \& Veit, Forthcoming).

Overall, it seems that medium levels of diversity do not necessarily translate into medium increases of concerns about immigration. Instead, they could result in effects that are of similar size as moving to high levels of diversity. Having said that, we should again consider the timing of cause and effect. During the anticipation period neither familiarization nor inter-group contact can level off threat effects, because both these moderators function via experiences that are only made once a person has moved. For this reason we expect initially (particularly during the anticipation period) stronger effects of moving to high diversity rather than to medium level diversity, but also expect these stronger effects to wear off over time.

\section{Data and measurement}

We test our hypotheses using the German Socio-Economic Panel Survey (SOEP), a panel survey with a yearly questionnaire since 1984 (Wagner, Frick, \& Schupp, 
2007). Under strict privacy conditions, the SOEP data can be connected with detailed neighborhood-level data that is provided by MICROM (Goebel, Spieß, \& Gerstenberg, 2007; Lersch, forthcoming, pp. 88-89). The MICROM data contains spatial data on the 'block' level, resulting in a unique data set containing geographical cells of as little as on average 460 households. The small size is an advantage, since especially the micro-neighborhood is found to be relevant for diversity effects (Dinesen \& Sønderskov, 2012). The blocks were originally defined by MICROM for geo-marketing purposes. Because of the availability of the MICROM-data, we restrict our analysis to the years 2008-2011. Overall, our sample contains 27,890 individuals and 78,311 person-year observations.

\section{Dependent variable}

Each survey year, respondents are asked what their main concerns are. The question is 'What is your attitude towards the following issues - are you concerned about them?' One of the issues is 'immigration to Germany' with the response categories 'very concerned' (32\%), 'somewhat concerned' (44\%), 'not concerned at all' (24\%). We recoded the variable into 'very concerned' (1) versus somewhat concerned / not concerned at all $(0)$.

Items capturing concern, or being worried about immigration are valid indicators to capture a negative attitude or feeling vis-à-vis immigration that is salient for the respondent (Fournier, Blais, Nadeau, Gidengil, \& Nevitte, 2003; McGhee \& Neiman, 2010). Accordingly, being concerned about immigration predicts center right-wing party identification (Pardos-Prado, Lancee, \& Sagarzazu, 2013). Wlezien (2005) shows that survey items measuring a public concern have two indistinguishable attributes: the salience of an issue and the degree to which it is seen as a problem. It is therefore plausible that respondents express both a salient and a negative attitude when they state to be very concerned about immigration.

\section{Independent variable}

Each year, respondents are asked whether they changed residence in the last year (yes/no). We combine this variable with the neighborhood information that we have for each respondent. Based on name analysis, MICROM provides the percentage of 
ethnic groups per block. The following groups are included: Germans, Turks, Spain/Portugal/Latin America, Italy, Greece, former Soviet Union, Eastern Europe, Balkan, Sub-Saharan Africa, South/East/South-East Asia, other non-European Islamic countries, Other. Based on these 12 groups, we calculated a Hirschman-Herfindahl Index (HHI) (Hirschman, 1964). The advantage of a name analysis is that it includes both nationals and non-nationals (such as naturalized citizens, or second and thirdgeneration immigrants), herewith describing the diversity of the neighborhood much better. Another advantage is its level of detail: it allows including multiple ethnic groups.

The disadvantage of the measure is that MICROM - for commercial reasons - does not provide much background information on how exactly the name analysis is carried out. We therefore correlated the percentage of persons of immigrant origin in the MICROM with the percentage of non-nationals from the (publicly available and official) Destatis data on the 'Kreise' level. Although Kreise are much larger than the MICROM units, this is the smallest geographical unit for which harmonized data is publicly available in Germany (Friedrichs \& Triemer, 2008). We hence collapsed our MICROM data to the Kreise level. The correlation is $0.87(\mathrm{p}<0.00)$, which suggests that MICROM is reliable.

We combine the moving information with the MICROM data on neighborhood diversity in the following way. First, we define the $15 \%$ most diverse MICROM blocks in Germany as high-diversity neighborhoods. Conversely, we define all blocks with levels of diversity that lie below the median $(50 \%)$ as homogeneous neighborhoods ${ }^{\mathrm{i}}$. All other blocks are medium-level diversity neighborhoods (above the median, but below the top $15 \%$ ). We define a change in diversity as a jump from one of these categories to another. This coding scheme allows us to look at both origin and destination effects. To make sure that people who experience only very small changes in HHI do not move categories, we additionally define a move to a different level of ethnic diversity as a change of at least half the inter-quartile range $\mathrm{e}^{\mathrm{ii}}$ (IQR) in the HHI compared to the origin neighborhood. We also considered other coding schemes to define changes in ethnic diversity, but this did not make a substantial difference. The coding scheme results in 270 moves from homogeneity to medium level diversity, 139 moves from homogeneity to high diversity, and 266 moves from medium-level diversity to high diversity. For moves to homogeneity, we only consider the second rule, that is, a 
move to homogeneity is a move that is followed by a reduction in the HHI of at least half the IQR (930 moves). The reason is that here we have no expectations regarding origin and destination effects. All other moves are treated as moves to equal levels of diversity (2,415 moves).

\section{Control variables}

On the individual level, we control for household type, labor market status, homeownership, and the number of previous moves. To control for potential selectivity in moving that is not captured by the DID-design (see below), we control with dummy variables for 13 reasons of moving (measured post-move). Reasons can be workrelated, family-related, a notice given by the landlord, financial, or that the respondent bought a new place. On the block level, we control for the average purchasing power, population density, and residential stability (all are measured by MICROM on a scale from one to nine).

\section{Empirical strategy}

To estimate the effect of moving, we make use of a differences-in-differences design (DID). The DID-design is often used to measure the causal effect induced by a particular event (the treatment) (Brüderl, 2005). By constructing a counterfactual (the control group) that is as similar as possible to the treatment group, one can estimate the effect of an event (Legewie, 2012). Veit (2015), Hémet (2015), and FisherWilliamson (2015) all use different, innovative designs to identify comparable treatment and control groups. In this paper we propose another strategy, which is particularly suited for panel data.

Yet, people who move are different from those who do not, particularly since people might move because they are upset about diversity. Therefore, the central idea of DID is not to compare movers to non-movers, but to compare the yearly trends in xenophobic attitudes of movers and non-movers (c.f. Angrist \& Pischke, 2009, Ch. 5). Movers might indeed hold different attitudes than non-movers. However, in the years that 'movers' do not move, the trend in xenophobia probably resembles that of nonmovers - irrespective of the different average levels of xenophobia that both groups hold. Insofar as the yearly trend of movers and non-movers is similar before the 
move, any change observed thereafter can be regarded as causal. Thus, one of the great advantages of the DID-estimator is that time-constant unobserved heterogeneity is no longer a problem. The DID-estimator is unbiased and consistent, even when the assumption that the unit effects are uncorrelated with the explanatory variable is violated. Furthermore, by respecting the causal ordering of events, reverse causality is unlikely. The DID-estimator is therefore often used to estimate causal effects (Halaby, 2004).

In this analysis, the event of moving is the treatment. In other words, moving to a different neighborhood implies that individuals are 'treated' with a change in diversity. The treatment effect represents the average change in anti-immigrant attitudes before and after the move, corrected for the general trend in attitudes for those individuals who did not move (the control group). The DID estimator is specified as follows:

$$
\mathrm{y}_{\mathrm{it}}=\alpha_{\mathrm{i}}+\lambda_{\mathrm{t}}+\delta \mathrm{d}_{\mathrm{it}}+\varepsilon_{\mathrm{it}}
$$

where $y$ is the outcome of interest of individual $i$ at time t. $\alpha$ is an individual-specific intercept that captures time-constant differences between all individuals. $\Lambda$ is a dummy variable that captures the yearly trends in $\mathrm{y}$, and $\mathrm{d}$ is a dummy variable that identifies person-time observations that have moved in the previous year. As such, $\delta$ captures the trend divergence of movers from the general yearly trends in y, i.e., it captures the difference in the yearly differences. $\varepsilon$ is the error term.

One of the fundamental assumptions of the DID estimator is the common trends assumption: the over-time (pre-move) trend in anti-immigrant attitudes should be the same for the control group (the non-movers) and the treatment group (the movers). If the (pre-move) trend in the outcome variable is similar for the two groups, this indicates that the groups are comparable. Consequently, differences between the groups that occur after the treatment can be interpreted as 'caused' by the treatment. To test the common trends assumption, following Angrist and Pischke (2009) we include yearly pre-treatment dummies for the movers. Statistically significant dummies indicate that the common trends assumption is not met: control and treatment group display different trends in attitudes towards immigrants before the event of moving.

Last, to find out whether the effect of moving persists or 'wears off' with time we estimate the effect separately for each year after the move. It is likely that the effect is 
strongest very close to the time of moving. As discussed in the theory section, it could even be that there is an anticipation effect. That is, it could be that attitudes already change (shortly) before the move (Bobo \& Zubrinsky, 1996). We therefore estimate the effect of moving half a year before the actual move, half a year after the move, and subsequently in yearly intervals. As a result, the DID-estimator is specified as follows:

$$
\mathrm{y}_{\mathrm{it}}=\alpha_{\mathrm{i}}+\lambda_{\mathrm{t}}+\delta_{-2} \mathrm{~d}^{(-2)}+\delta_{-1} \mathrm{~d}^{(-1)}+\delta_{1} \mathrm{~d}^{(1)}+\delta_{2} \mathrm{~d}^{(2)}+\mathrm{X}_{\mathrm{it}} \beta+\varepsilon_{\mathrm{it}}
$$

, which we estimate as a two-way fixed-effects equation.

\section{Results}

Figure 3.1 visualizes the main findings: On the left-hand side, individuals who moved to diversity, on the right-hand side those who moved to equal diversity or homogeneity. At $y=0$, the $x$-axis (black line) represents the trend for the non-movers. More specifically, non-movers are people who did not move in the survey year, will not move in the next two years, and have not moved in any of the previous years. Accordingly, the figure only shows the divergence from the general trend in concerns about immigration (2008-2011). The visualization of Figure 3.1 hence conceals that, overall, concerns about immigration decreased in the observed years. Compared to 2008, nonmovers were less concerned about immigration in the following years: In 2009, 4 per cent of the sample changed their attitude from being very concerned about immigration to not being concerned at all. In 2011, this number is 2.9 per cent. Theoretically, these trends are not very meaningful, but they represent the baseline against which we compare the change in immigration concerns of movers. The $\mathrm{x}$-axis represents the time relative to the event of moving, the vertical dashed line identifies the moving event, and lastly, the grey box acknowledges that moving is not an unanticipated accident but a planned transition, i.e., people know that they will move in the next months and they probably also know where they will move to. Finally, the y-axis is the divergence from the general trend in the probability to be very concerned about immigration.

In principle, the estimates for our control variables (not shown here) are also interesting since they are rare longitudinal evidence from two-way fixed effects models. 
Note, however, that in such a set-up, contextual neighborhood effects might be too conservative since a neighborhood's average purchasing power, for example, does not change much in one year. With hardly any changes on the contextual level, it comes at no surprise that neither purchasing power (as a measure of neighborhood SES), nor population density or residential instability are associated with concern about immigration. Our analysis of moving to diversity does not suffer from this problem, because we defined moving to diversity in such a way that movers experienced a substantial increase in diversity (at least half the inter-quartile range). Against this background, we focus on our main research questions and analysis.

Figure 3.1 shows that the pre-treatment dummies are not statistically significant. This means that trends in concern about immigration of people who will move in the next one or two years (the treatment group) are not significantly different from non-movers (the control group). In other words, the common trends assumption is not violated and

Figure 3.1: The effect of moving on being very concerned about immigration.

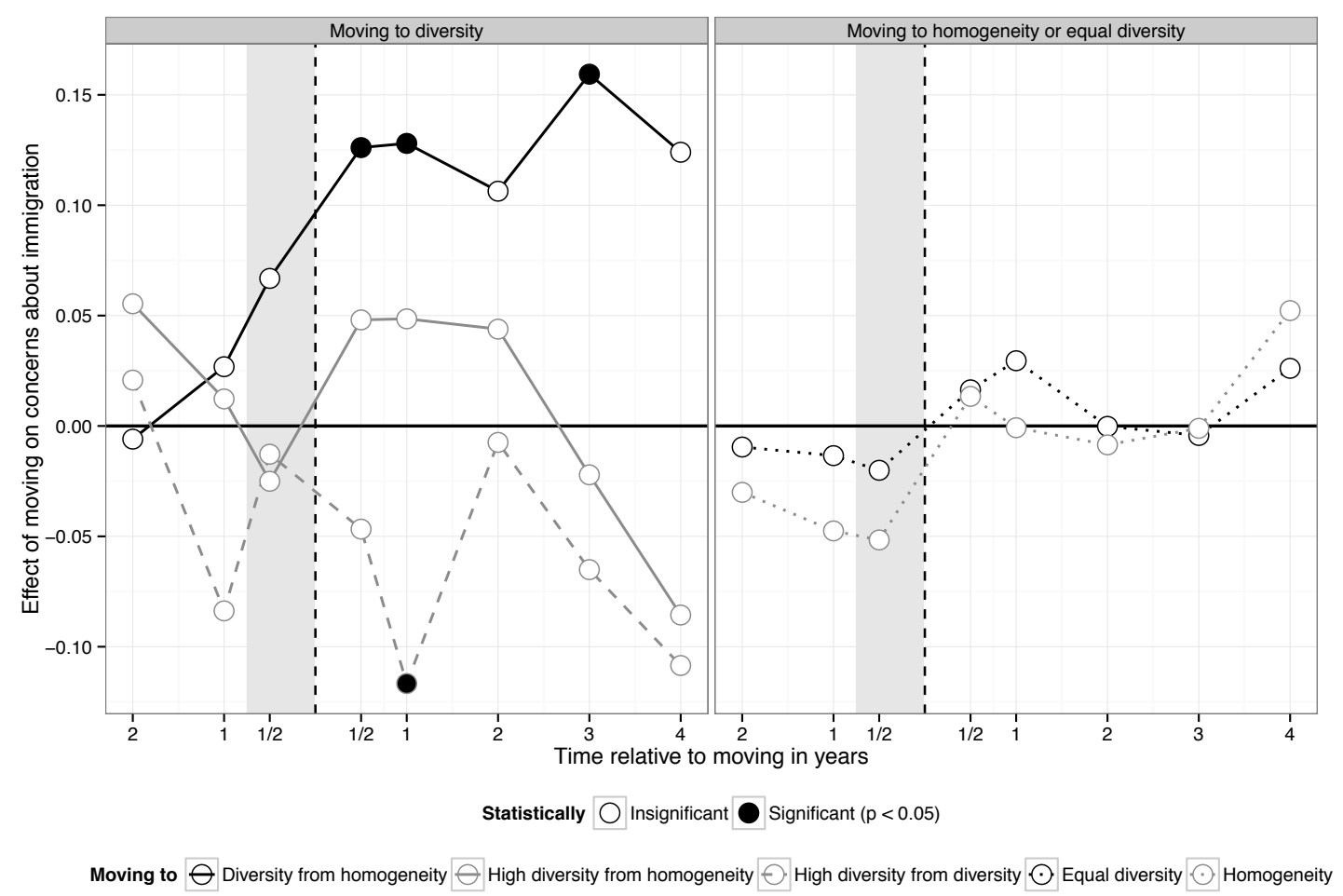

in principle assures that any significant differences after persons have moved can be interpreted as causal. Apart from looking at significance levels only, the assumption seems best met for people who move to medium-level diversity. The trends of those 
who move to high levels of diversity look somewhat different, even though these differences are insignificant.

Yet, against the common trends assumption, the pre-treatment dummies also show that six months or less before the move, those individuals who are moving to diversity are slightly (but not statistically significantly) more concerned about immigration than non-movers. In other words, shortly before people move to medium-level diversity, the common trends start to diverge. Yet, this expected anticipation effect is not statistically significant, even though the trend continues after the move and eventually becomes significant.

For people who move to medium levels of diversity, we see an increase in concern about immigration. More than 13 per cent of movers become concerned about immigration directly after they have moved. That is, when individuals are confronted with increasing diversity, perceived threat increases, resulting in more negative attitudes towards immigration. What is more, this effect does not wear off; we do not see a short time shock with a quick adaptation. In Figure 3.1, it is also visible that the effect of moving to diversity persists over time: for the years after moving, people who moved to diversity are more xenophobic than they were before and compared to nonmovers.

For two time points, the coefficient is not statistically significant at the $5 \%$ level (with p-values of .08 and .09 in a two-tailed test). Yet, The $\mathrm{N}$ of movers is relatively low. Furthermore, if one would assume a directed hypothesis, these time points are significant on a 5\% level. Last, the effect size remains substantial, that is, more than 10 per cent (after two years) remain concerned throughout the first four years after moving and none of our results suggest a leveling off after that period. Indeed, if one estimates a model without specifying separate years after moving, the effect of moving to diversity is significant In conclusion, it seems that even four years after moving, neither familiarization with diversity, nor inter-ethnic contacts balance threat effects.

Surprisingly, for people who move to high levels of diversity we do not observe changes in their concern about immigration. Theoretically, for people who move to high diversity we expected strong anticipated threat effects, which level off after the move because of familiarization (Schneider, 2008) and opportunities for inter-ethnic contact (Schaeffer, 2013). Instead, we find no anticipation effects, irrespective of 
whether people come from diversity or from homogeneity. This offsetting finding continues as a trend, that is, feelings of threat do not occur after people move to high diversity, either.

Last, people who move to homogeneity, or to equal levels of diversity do not significantly change their concerns about immigration. It seems that, despite the reduced competition over resources, people stick to their political opinion about immigration when they settle in a more homogeneous neighborhood.

\section{Robustness check: threat-immune movers?}

While our analytical strategy is thorough and excludes bias from unobserved heterogeneity, the null-results about moving to high diversity are partly implausible. Perhaps people who move to high levels of diversity are immune to diversity-induced threat, just like men are immune to the contraceptive pill, whether randomly treated in a double blind experiment or not. Put differently, in a DID-design, we estimate the Average Treatment effect for the Treated (ATT) (Morgan \& Winship, 2007), but who are the treated? Based on the literature on determinants of xenophobia (Ceobanu \& Escandell, 2010), there are five arguments why people might be immune for diversity-induced threat. First, people who move to high diversity could be dominantly immigrants and for them, co-ethnics, or other immigrants are less likely to be a threat. Second, people who move to high diversity might have so many close intergroup friends that they are threat immune. Third, it could be that such movers are all highly educated full-time workers who do not fear any competition from immigrants. Fourth, people who move to high diversity might be frequent movers who are not affected by their neighborhood because they stay only shortly. Finally, there could be a ceiling effect: movers might hold such negative attitudes that their concerns cannot increase any further. Conversely, one might expect people who move to high diversity to favor cultural richness and to hold such positive attitudes about immigrants that they are threat immune. Here too we would expect differences in pre-move levels of xenophobia.

To empirically test these explanations, Figure 3.2 shows estimates of a multinomial logistic regression predicting the likelihood to move to high diversity (from homogeneity or diversity respectively) as compared to medium-level diversity as afunction of the above-mentioned determinants of xenophobia. This comparison of diversity mov- 
ers shows that there are indeed significant differences with regard to having an immigrant origin. Both first- and second-generation immigrants are more likely to move to high diversity from diversity and less likely to move to medium level diversity or high diversity from homogeneity. In contrast to previous research we do not see any differences in terms of pre-move levels of xenophobia (i.e., xenophobic people are not less likely to move to high diversity than to medium level diversity), nor in terms of education, being subject to labor market competition (unemployed, in education or vocational training as compared to being employed or inactive), the frequency of moving, or having intergroup friends.

Figure 3.2: Threat-immune individuals? Multi-nominal regression predicting the likelihood to move to high diversity as compared to medium level diversity.

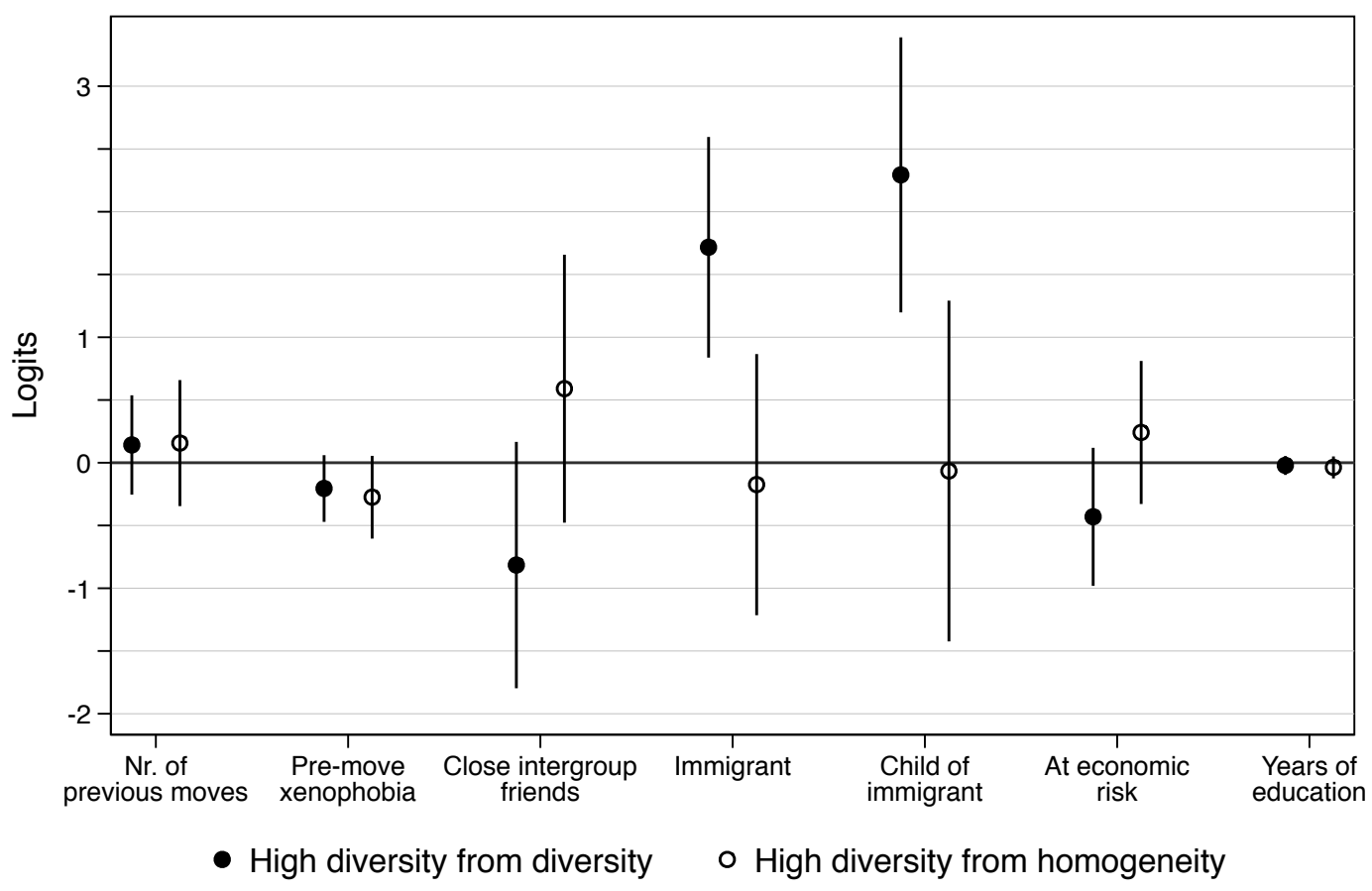

Does being of immigrant origin explain the null findings of moving to high diversity? In Figure 3.3, we present estimates from a similar DID set-up, but excluding individuals of migrant origin (both first and second generation). In other words, we exclude the potentially 'threat-immune' people from the analysis. As can be seen in Figure 3.3, the overall pattern remains. 
Figure 3.3. Sensitivity analysis for the effect of moving on being very concerned about immigration.

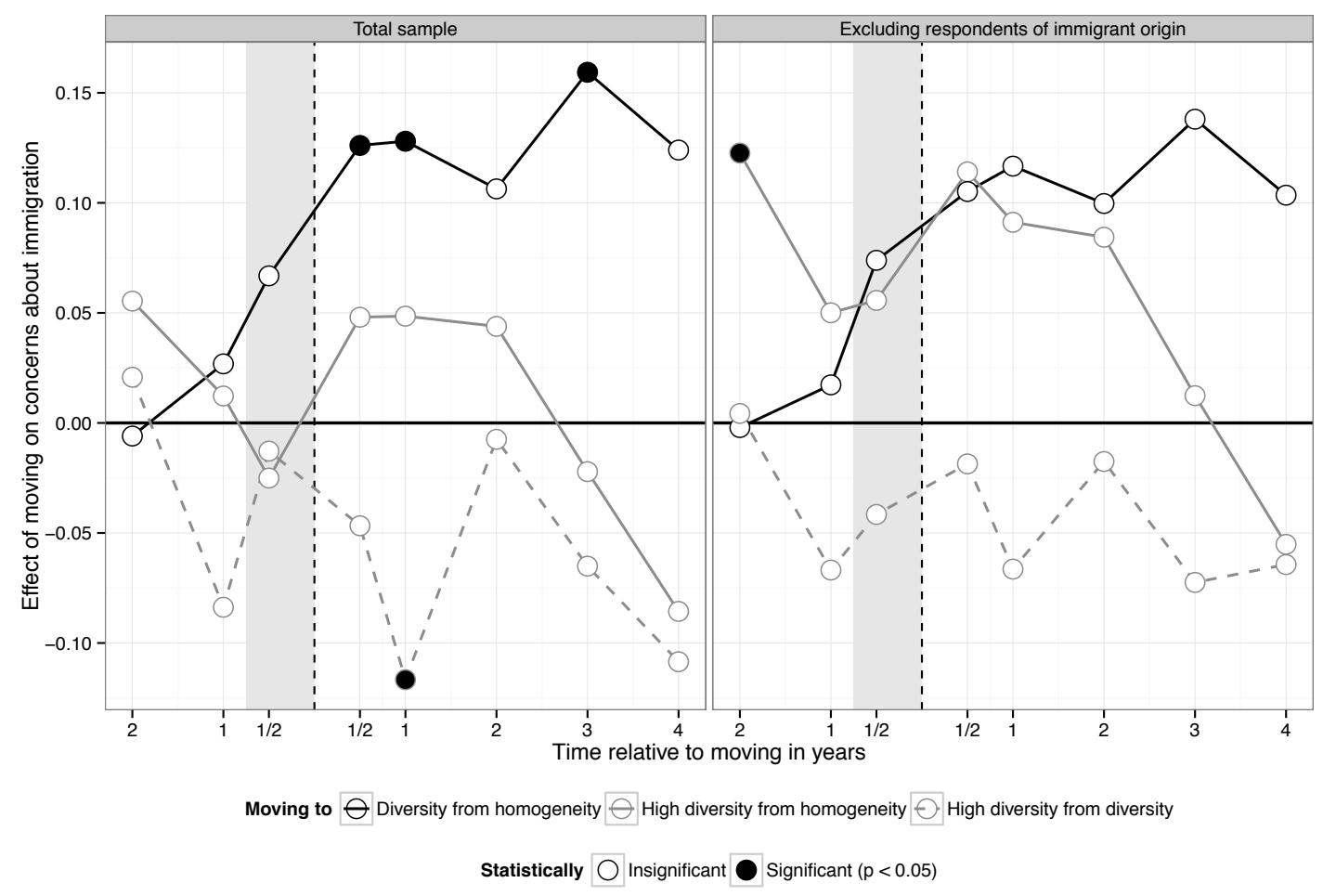

The reduced sample size implies a loss of statistical power, but the pattern of increased xenophobia after moving to medium-level diversity remains and does not wear off either. Furthermore, the estimates that lose significance still have a p-value below 10 per cent. Excluding the potentially threat-immune population does not change the results for moves to high diversity in statistically significant ways, either. Although estimates for moves to medium and high diversity look similar up to two years after the move, the coefficients of the latter have p-values larger than 0.7 and are thus far from meeting any criteria of statistical significance. Rather, the results question the validity of the common-trends assumption for persons moving to high diversity. All in all, this robustness check questions the orthodox assumption that persons of immigrant origin are a diversity-threat-immune population. If they were, we should see an increase in the negative response to diversity. Instead, the results remain virtually unchanged, suggesting that persons of immigrant origin are also subject to negative diversity effects, just like majority group members. The case numbers are too low for a direct inference-statistical test of this conclusion. But additional results indeed suggest that after a move to medium diversity concerns about immigration increase as much (if not more) for persons of immigrant origin as they do for natives. ${ }^{\text {iii }}$ This interpretation is corroborated by Veit's (2015) experimental study. 
In summary, the robustness check supports a causal interpretation of the effect of moving to medium-level diversity. Yet, the robustness check did not solve the puzzle of the null-results for moving to high diversity. Last, the robustness check suggests that also for persons of immigrant origin concerns about immigration increase as they move to more diverse neighborhoods. Indeed, if we restrict the sample to immigrants only, the point estimates for moving to diversity are even larger, but due to the small sample size, none of the coefficients are statistically significant.

\section{Conclusion}

In this paper, we made use of longitudinal data to analyze to what extent people's attitudes toward immigration change when they move to a more diverse neighborhood. Generally, our findings corroborate negative ethnic diversity effects, that is, we show that individuals who move to a more diverse neighborhood are about $13 \%$ more likely to become very concerned about immigration, suggesting that increasing diversity operates as a trigger for (perceived) ethnic threat, rather than for an increase in contact. Because individuals who move to equal or lower diversity neighborhoods do not change their attitudes, it is not the event of moving as such that makes people more negative towards out-groups, but the change towards a more diverse environment. By applying a differences-in-differences design, we also methodologically improved upon previous work. Our longitudinal set-up not only excludes reverse causality, but also renders unobserved heterogeneity a very unlikely alternative explanation. Beyond the general finding of a negative effect of diversity, our results offer further insights. First, we are able to show that the negative effect of diversity is not a shortterm shock that wears off soon after adapting to the new environment. We observe an increase of concerns over immigration that remains even three years after the move. This implies that adaptation processes that attenuate cognitive biases take quite a long time. Alternatively, it could mean that the mechanisms behind negative diversity effects are more complex, including cooperation problems or incongruent preferences, as laid out in the introduction to this volume. Such obstacles are of course not overcome by individual adaptation.

This study is the first to investigate whether previous experiences with diversity eliminate any threat effects of moving to a more diverse place. Interestingly, our results 
lend no consistent support for this hypothesis: while it is only people who come from homogeneous neighborhoods that become more concerned about immigration, those who move from homogeneity to high levels of diversity do not change their views. We also investigated destination effects and expected that moving to high levels of diversity rather than to medium level diversity should result in initially larger concerns about immigration that wear off once familiarization and inter-ethnic contact experiences set it. Surprisingly, however, we found no statistically significant reactions of people moving to highly diverse neighborhoods whatsoever. We considered whether the null-effect for those moving to the top 15 per cent most diverse neighborhoods might be due to 'threat immunity' of such movers. Our sensitivity analyses questions this objection. Instead, it suggests that populations often thought to be immune to threat might respond similar to the majority population. That is, persons of immigrant origin equally react with growing concerns over immigration when they move to more diverse neighborhoods.

One should keep in mind that these findings are specific to the levels of diversity that we observed. Germany's 'very high' diversity is fairly moderate compared to other countries. In Germany, the cut-off point for the top 15 per cent most diverse neighborhoods is at $\mathrm{HHI}=0.21$. This is certainly low in terms of the range that is analytically possible. Similarly, it is important to keep in mind that the event of moving to diversity is not the same as changing diversity of the neighborhood. Moving involves a large degree of choice and people that do move represent a certain part of the general population. Homeowners probably react in a more extreme way to a change of their environment that lies beyond their control. When moving to diversity increases concerns about immigration, it is likely that the level of diversity in the neighborhood a person lives in changing has an even stronger effect on people's attitudes. In other words, our test of the diversity hypothesis is a very conservative one. Unfortunately, our data did not allow us to test the effect of a changing neighborhood. In the four years of survey data that we had at our disposal, the diversity of neighborhoods changed very little.

Future analyses could focus on replication of the current results in different contexts, such as, for example, the US. Insofar as future studies continue to find no threat effects for those moving to high levels of diversity, social scientists have another puzzle to solve. Furthermore, future analyses will need to show whether our findings equally 
hold true for other outcome variables that are relevant in the study of social cohesion, such as trust, cooperative behavior, and collective action. 


\section{References}

Angrist, J. D. \& Pischke, J.-S. (2009). Mostly harmless econometrics. Princeton: Princeton University Press.

Bobo, L. \& Zubrinsky, C. L. (1996). Attitudes on Residential Integration: Perceived Status Differences, Mere In-Group Preference, or Racial Prejudice? Social Forces, 74(3), 883-909.

Brüderl, J. (2005). Panel data anlysis. http://www2.sowi.unimannheim.de/lsssm/veranst/Panelanalyse.pdf

Ceobanu, A. M. \& Escandell, X. (2010). Comparative Analyses of Public Attitudes Toward Immigrants and Immigration Using Multinational Survey Data: A Review of Theories and Research. Annual Review of Sociology, 36(1), 309328.

Clark, W. V. (1992). Residential preferences and residential choices in a multiethnic context. Demography, 29(3), 451-466.

Crowder, K. (2000). The Racial Context of White Mobility: An Individual-Level Assessment of the White Flight Hypothesis. Social Science Research, 29(2), 223-257.

Crowder, K., Hall, M., \& Tolnay, S. E. (2011). Neighborhood Immigration and Native Out-Migration. American Sociological Review, 76(1), 25-47.

de Souza Briggs, X. (1998). Brown kids in white suburbs: Housing mobility and the many faces of social capital. Housing Policy Debate, 9(1), 177-221.

Dinesen, P. T. \& Sønderskov, K. M. (2012). Trust in a Time of Increasing Diversity: On the Relationship between Ethnic Heterogeneity and Social Trust in Denmark from 1979 until Today. Scandinavian Political Studies, 35(4), 273294.

Dixon, J. C. (2006). The Ties That Bind and Those That Don't: Toward Reconciling Group Threat and Contact Theories of Prejudice. Social Forces, 84(4), 21792204.

Eric Oliver, J. \& Wong, J. (2003). Intergroup Prejudice in Multiethnic Settings. American Journal of Political Science, 47(4), 567-582. 
Fisher Williamson, A. (2015). Declining trust amid diversity? A natural experiment in Lewiston, Maine. In R. Koopmans, B. Lancee and M. Schaeffer (Eds.): Social Cohesion and Immigration in Europe and North America: Mechanisms, Conditions, and Causality. London: Routledge, 56-84

Fournier, P., Blais, A., Nadeau, R., Gidengil, E., \& Nevitte, N. (2003). Issue Importance and Performance Voting. Political Behavior, 25(1), 51-67.

Friedrichs, J. \& Triemer, S. (2008). Gespaltene Städte? Soziale und ethnische Segregation in deutschen Großstädten. Wiesbaden: VS Verlag für Sozialwissenschaften.

Goebel, J., Spieß, C. K., \& Gerstenberg, S. (2007). Die Verknüpfung des SOEP mit MICROM-Indikatoren: Der MICROM-SOEP Datensatz. Berlin DIW.

Gundelach, B. \& Freitag, M. (2013). Neighbourhood Diversity and Social Trust: An Empirical Analysis of Interethnic Contact and Group-specific Effects. Urban Studies.

Halaby, C. N. (2004). Panel models in sociological research. Annual Review of Sociology, 30, 507-544.

Harris, D. R. (2001). Why Are Whites and Blacks Averse to Black Neighbors? Social Science Research, 30(1), 100-116.

Hémet, C. (2015). Diversity and well-being: local effects and causal approaches. In R. Koopmans, B. Lancee and M. Schaeffer (Eds.): Social Cohesion and Immigration in Europe and North America: Mechanisms, Conditions, and Causality. London: Routledge, 23-37

Hirschman, A. O. (1964). The Paternity of an Index. The American Economic Review, 54(5), 761-762.

Hopkins, D. J. (2009). The Diversity Discount: When Increasing Ethnic and Racial Diversity Prevents Tax Increases. The Journal of Politics, 71(01), 160-177.

Hopkins, D. J. (2010). Politicized Places: Explaining Where and When Immigrants Provoke Local Opposition. American Political Science Review, 104(1), 40-60. 
Jackson, M. I. \& Mare, R. D. (2007). Cross-sectional and longitudinal measurements of neighborhood experience and their effects on children. Social Science Research, 36(2), 590-610.

Kang, N. \& Kwak, N. (2003). A Multilevel Approach to Civic Participation: Individual Length of Residence, Neighborhood Residential Stability, and Their Interactive Effects With Media Use. Communication Research, 30(1), 80-106.

Koopmans, R.; Lancee, B. \& Schaeffer M. (2015). Ethnic diversity in diverse societies: an introduction. In R. Koopmans, B. Lancee and M. Schaeffer (Eds.): Social Cohesion and Immigration in Europe and North America: Mechanisms, Conditions, and Causality. London: Routledge, 1-20

Koopmans, R. \& Veit, S. (Forthcoming). Ethnic Diversity, Trust, and the Mediating Role of Positive and Negative Interethnic Contact: A Priming Experiment. Social Science Research.

Lancee, B. \& Pardos-Prado, S. (2013). Group Conflict Theory in a Longitudinal Perspective: Analyzing the Dynamic Side of Ethnic Competition. International Migration Review, 47(1), 106-131.

Legewie, J. (2012). Die Schätzung von kausalen Effekten: überlegungen zu Methoden der Kausalanalyse anhand von Kontexteffekten in der Schule. KZfSS Kölner Zeitschrift für Soziologie und Sozialpsychologie, 64(1), 123-153.

Lersch, P. M. (forthcoming). Residential Relocations and Their Consequences. Life course effects in England and Germany: Springer.

Lin, K.-C., Twisk, J. W. R., \& Rong, J.-R. (2011). Longitudinal Interrelationships Between Frequent Geographic Relocation and Personality Development: Results From the Amsterdam Growth and Health Longitudinal Study. American Journal of Orthopsychiatry, 81(2), 285-292.

Martinovic, B., van Tubergen, F., \& Maas, I. (2009). Dynamics of Interethnic Contact: A Panel Study of Immigrants in the Netherlands. European Sociological Review, 25(3), 303-318.

McGhee, E. M. \& Neiman, M. (2010). Concern over immigration and support for public services. California Journal of Politics and Policy, 2(1), 1-26. 
Meuleman, B., Davidov, E., \& Billiet, J. (2009). Changing attitudes toward immigration in Europe, 2002-2007: a dynamic group conflict approach. Social Science Research, 38, 352-365.

Morgan, S. L. \& Winship, C. (2007). Counterfactuals and Causal Inference: Methods and Principles for Social Research. Cambridge: Cambridge University Press.

Nisic, N., \& Petermann, S. (2013). New City= New Friends? The Restructuring of Social Resources after Relocation. Comparative Population Studies-Zeitschrift für Bevölkerungswissenschaft, 38(1), 199-226.

Oishi, S. (2010). The Psychology of Residential Mobility: Implications for the Self, Social Relationships, and Well-Being. Perspectives on Psychological Science, $5(1), 5-21$.

Oishi, S., Rothman, A. J., Snyder, M., Su, J., Zehm, K., Hertel, A. W., \& Sherman, G. D. (2007). The socioecological model of procommunity action: the benefits of residential stability. Journal of Personality and Social Psychology, 93(5), 831.

Olzak, S. (1992). The Dynamics of Ethnic Competition and Conflict. Stanford Stanford University Press.

Pardos-Prado, S., Lancee, B., \& Sagarzazu, I. a. (2013). Immigration and Electoral Change in Mainstream Political Space. Political Behavior, 1-29.

Pettit, B. \& McLanahan, S. (2003). Residential Mobility and Children's Social Capital: Evidence from an Experiment. Social Science Quarterly, 84(3), 632649.

Sampson, R. J. (1988). Local Friendship Ties and Community Attachment in Mass Society: A Multilevel Systemic Model. American Sociological Review, 53(5), 766-779.

Schaeffer, M. (2013). Which groups are mostly responsible for problems in your neighbourhood? Ethnic and Racial Studies, 36(1), 156-178.

Schaeffer, M. (2014). Ethnic Diversity and Social Cohesion: Immigration, Ethnic Fractionalization and Potentials Civic Action. Aldershot: Ashgate.

Schlueter, E. \& Scheepers, P. (2010). The relationship between outgroup size and anti-outgroup attitudes: A theoretical synthesis and empirical test of group 
threat- and intergroup contact theory. Social Science Research, 39(2), 285295.

Schlueter, E. \& Wagner, U. (2008). Regional Differences Matter: Examining the Dual Influence of the Regional Size of the Immigrant Population on Derogation of Immigrants in Europe. International Journal of Comparative Sociology, 49(23), 153-173.

Schneider, S. (2008). Anti-Immigrant Attitudes in Europe: Outgroup Size and Perceived Ethnic Threat. European Sociological Review, 24(1), 53-67.

Semyonov, M., Raijman, R., \& Gorodzeisky, A. (2006). The Rise of Anti-Foreigner Sentiment in European Societies, 1988-2000. American Sociological Review, $71(3), 426-449$.

Semyonov, M., Raijman, R., Tov, A. Y., \& Schmidt, P. (2004). Population size, perceived threat, and exclusion: a multiple-indicators analysis of attitudes toward foreigners in Germany. Social Science Research, 33(4), 681-701.

Stokols, D., \& Shumaker, S. A. (1982). The Psychological Context of Residential Mobility and Weil-Being. Journal of Social Issues, 38(3), 149-171.

Uslaner, E. 92015). Diversity, segregation, and trust. In R. Koopmans, B. Lancee and M. Schaeffer (Eds.): Social Cohesion and Immigration in Europe and North America: Mechanisms, Conditions, and Causality. London: Routledge, 87-104

Veit, S. (2015). Thinking about ethnic diversity: experimental evidence on the causal role of ethnic diversity in German neighbourhoods and schools. In R. Koopmans, B. Lancee and M. Schaeffer (Eds.): Social Cohesion and Immigration in Europe and North America: Mechanisms, Conditions, and Causality. London: Routledge, 167-186

Wagner, G., Frick, J., \& Schupp, J. R. (2007). The German Socio-Economic Panel study (SOEP)-evolution, scope and enhancements. Schmollers Jahrbuch, 127(1), 139-169.

Wlezien, C. (2005). On the salience of political issues: The problem with 'most important problem'. Electoral Studies, 24(4), 555-579. 


\section{Notes}

${ }^{i}$ When we use lower cut-off values to define homogeneity, our results remain similar but because of low case numbers they drop from significance.

ii The inter-quartile range, also called the midspread or middle fifty, is a measure of statistical dispersion, equal to the difference between the upper and lower quartiles. In case a variable is skewed, the IQR is a better measure of dispersion than, for example, the standard deviation.

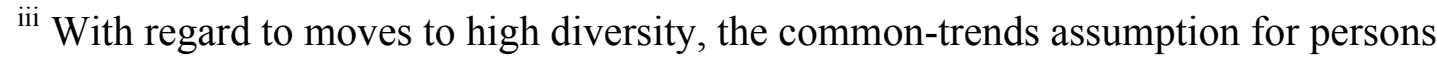
of immigrant origin is strongly violated and thus the results are inconclusive. 